\section{Reconstruction of Reflectance Spectra Using Robust Nonnegative Matrix Factorization}

A. Ben Hamza and David J. Brady

\begin{abstract}
In this correspondence, we present a robust statistics-based nonnegative matrix factorization (RNMF) approach to recover the measurements in reflectance spectroscopy. The proposed algorithm is based on the minimization of a robust cost function and yields two equations updated alternatively. Unlike other linear representations, such as principal component analysis, the RNMF technique is resistant to outliers and generates nonnegative-basis functions, which balance the logical attractiveness of measurement functions against their physical feasibility. Experimental results on a spectral library of reflectance spectra are presented to illustrate the much improved performance of the RNMF approach.
\end{abstract}

Index Terms-Nonnegative matrix factorization, reflectance spectra, robust statistics.

\section{INTRODUCTION}

Reflectance spectroscopy is the study of light as a function of wavelength that has been emitted, reflected, or scattered from a solid, liquid, or gas. These functions, also called spectra, provide useful information about the chemical composition of a material, and are a powerful tool for identifying the elements of a material by examining the spectrum of their radiation [1]. Collection and analysis of spectra typically involves a source of light (or other electromagnetic radiation), an element to separate the light into its component wavelengths, and a detector to sense the presence of light after separation of wavelengths. Reflectance spectroscopy can be used to derive significant information about mineralogy and with little or no sample preparation. It may be used in applications when other methods would be too time consuming. For example, imaging spectrometers are already acquiring millions of spatially gridded spectra over an area from which mineralogical maps are being made. The reflectance spectroscopy of minerals will be the focus of this correspondence, but the results may apply to any other material.

In this correspondence, we propose a robust statistics-based approach to nonnegative matrix factorization of a reflectance spectral library. The approach is formulated as a robust optimization problem whose solution is achieved using the method of steepest descent and yields two equations updated alternatively. Unlike the Karhunen-Loeve (K-L) method [2], the nonnegative matrix factorization technique [4], [5] generates nonnegative basis functions, which balances the logical attractiveness of measurement functions against their physical feasibility.

The rest of this correspondence is organized as follows. The next section is devoted to the problem formulation, a brief introduction to $\mathrm{K}-\mathrm{L}$ approximation, and also a description of the nonnegative matrix factorization technique. In Section III, we propose a robust nonnegative matrix factorization (RNMF) approach to recover reflectance spectra. This approach is based on the minimization of a robust statistics-based

Manuscript received April 11, 2005; revised November 1, 2005. The associate editor coordinating the review of this manuscript and approving it for publication was Dr. Ercan E. Kuruoglu.

A. B. Hamza is with the Concordia Institute for Information Systems Engineering, Concordia University, Montréal, QC H3G 1T7 (e-mail: hamza@ ciise. concordia.ca).

D. J. Brady is with the Department of Electrical and Computer Engineering, Duke University, Durham, NC 27707 USA (e-mail: dbrady@ee.duke.edu).

Digital Object Identifier 10.1109/TSP.2006.879282 energy function and yields two equations based on additive rules that are updated alternatively. In Section IV, we provide numerical simulations to show the power of the RNMF technique in the reconstruction of reflectance spectra, and we also provide a comparative study with other related methods proposed in the literature. Finally, Section V provides some conclusions.

\section{SENSING AND APPRoximation}

\section{A. Karhunen-Loeve Approximation}

A basic linear technique in dimensionality reduction is $\mathrm{K}-\mathrm{L}$ decomposition, also known as principal component analysis (PCA), which calculates a set of basis vectors that can be used to approximate high-dimensional data optimally in the least-squares sense [2]. The number of basis vectors is much smaller than the number of dimensions, so encoding the data as linear combinations of the basis vectors transforms it to a lower dimensional space. This dimension reduction can be used to improve for explanation, visualization, and features extraction. PCA is an explanatory technique to learn about datasets. The objective of PCA to reduce the dimensionality of the data set while retaining as much as possible the variation in the data set. Principal components are linear transformations of the original set of variables and are uncorrelated and ordered so that the first few components carry most of the variations in the original data set. The first principal component has the geometric interpretation that it is a new coordinate axis that maximizes the variation of the projections of the data points on the new coordinate axis. Given a data set of signals $S=\left\{\boldsymbol{f}_{j}: j=1, \ldots, n\right\}$ where each $\boldsymbol{f}_{j}$ is an $m$-dimensional vector, the PCA is a linear projection technique

$$
\boldsymbol{y}_{j}=E_{d}^{T}\left(\boldsymbol{f}_{j}-\overline{\boldsymbol{f}}\right), \quad \forall j=1, \ldots, n
$$

where $\boldsymbol{y}_{j}$ is a $d$-dimensional projected data vector (usually $d \ll m$ ), $\boldsymbol{f}_{j}$ is the original data vector, $\overline{\boldsymbol{f}}$ is its sample mean i.e., $\bar{f}=\left(\sum_{j=1}^{n} \boldsymbol{f}_{j}\right) / n$, and $E_{d}^{T}$ is a $d \times m$ matrix that contains the PCA projection vectors. The $d$-projection vectors that maximize the variance of the principal axes $\boldsymbol{y}_{j}$ are given by the eigenvectors of the matrix $\Sigma=S S^{T}$. The matrix commonly used in finding such eigenvectors is the covariance matrix, which would coincide with the matrix $\Sigma$ in the case of a null sample mean [3].

Reconstruction techniques must balance the logical attractiveness of measurement functions against their physical feasibility. In imaging and spectrometry, all measurements at the optical-electronic are strictly positive photon counts. Unfortunately, the $\mathrm{K}-\mathrm{L}$ decomposition does not generate strictly positive basis functions. This means that it may not be possible to directly sample principal components or that such measurement may require more complex embedded bipolar electronic processing. With a view to avoiding this problem, we consider the use of nonnegative matrix factorization to generate a nonnegative measurement basis. This approach is described in the next subsection.

\section{B. Nonnegative Matrix Factorization}

One major problem with PCA is that the basis vectors may have both positive and negative components, and the data are represented as linear combinations of these vectors with positive and negative coefficients. The optimality of PCA can be traced back to construction cancellation of the signs. In many applications, the negative components contradict physical realities. For example, the pixels in a grayscale image have nonnegative intensities, so an image with negative intensities cannot be reasonably interpreted. To address this problem, a recent approach called nonnegative matrix factorization (NMF) was proposed to search 
for a representative basis with only nonnegative vectors [4], [5]. In other words, the idea of NMF may be interpreted as factorizing a nonnegative data matrix into two nonnegative matrices. The NMF approach can be formulated as follows. Given a nonnegative data matrix of signals $S$ of size $m \times n$, where $m$ is the length of each signal and $n$ is the number of signals (i.e., each column of $S$ is a signal), we can approximately factorize $S$ into nonnegative matrices $W$ and $H$ with sizes $m \times r$ and $r \times n$, respectively, that is $S \approx W H$. The desired number $r$ is usually chosen to be smaller than $m$ or $n$, so that the rank of $W$ and $H$ is smaller than $S$, and therefore this yields a compressed version of the original data matrix $S$. The matrix $W$ contains the NMF basis vectors, and the matrix $H$ contains the associated coefficients (nonnegative weights). In vector form, we can write $S \approx W H$ as $f_{j} \approx W \boldsymbol{h}_{j}$, where $f_{j}$ and $\boldsymbol{h}_{j}$ are the columns of $S$ and $H$, respectively, and $j=1, \ldots, n$. Therefore, each signal $\boldsymbol{f}_{j}$ is approximated by a linear combination of the columns of $W$, weighted by the components of $\boldsymbol{h}_{j}$. Therefore, $W$ can be viewed as containing a basis that is optimized for the linear approximation of the spectra in $S$.

To measure the quality of the approximation factorization $S \approx W H$, a cost function between $S$ and $W H$ needs to be optimized subject to nonnegativity constraints on $W$ and $H$. This is done by minimizing the least-squares cost function given by

$$
\|S-W H\|^{2}=\sum_{i j}\left(S_{i j}-(W H)_{i j}\right)^{2} .
$$

The minimization of (1) yields the following multiplicative update rules at the $t$ th iteration [5]:

$$
\begin{aligned}
W_{i k}^{(t+1)} & =W_{i k}^{(t)} \frac{\left(S H^{T}\right)_{i k}^{(t)}}{\left(W H H^{T}\right)_{i k}^{(t)}} \quad \text { and } \\
H_{k j}^{(t+1)} & =H_{k j}^{(t)} \frac{\left(W^{T} S\right)_{k j}^{(t)}}{\left(W^{T} W H\right)_{k j}^{(t)}}
\end{aligned}
$$

where the matrices $W$ are $H$ are initialized as nonnegative random matrices, and the updates are done alternatively, that is after updating one row of $H$, we need to update the corresponding column of $W$. In other words, we should not update the whole matrix $H$ first followed by an update of the matrix $W$. The NMF algorithm is therefore an iterative optimization algorithm, which modifies at each iteration the nonnegative basis functions (i.e., columns of $W$ ) and encodings (i.e., $H_{k j}$ ) until convergence. Given a signal $\boldsymbol{f}_{j}=W \boldsymbol{h}_{j}$, where $\boldsymbol{f}_{j}$ and $\boldsymbol{h}_{j}$ are the columns of $S$ and $H$, respectively, the reconstructed spectrum $\hat{\boldsymbol{f}}_{j}$ is given by $\hat{\boldsymbol{f}}_{j}=W \hat{\boldsymbol{h}}_{j}$, where $\hat{\boldsymbol{h}}_{j}$ is obtained by minimizing $\left\|\boldsymbol{W} \boldsymbol{h}_{j}-\boldsymbol{f}_{j}\right\|^{2}$ subject to $\boldsymbol{h}_{j} \geq 0$, which is referred to as linear least squares with nonnegativity constraints.

\section{PROPOSED METHOD}

The goal of the proposed nonnegative matrix factorization method is to obtain basis vectors that are not influenced by outliers. Unfortunately, the least squares method given by (1) and (2) produces estimates that are sensitive to outliers. In recent years, a great deal of attention has been focused on robust statistics-based methods [12], [13]. These robust techniques have been successfully applied to a variety of imaging and computer vision applications [14]-[17] and produce results that are resistant to the presence of outliers. The idea behind robust statistics methods is to replace the least-squares criterion in (1) by another criterion that is less sensitive to the presence of outliers. Recently Samson et al. [10] introduced a robust hypersurface cost function $\rho$ defined as

$$
\rho(\|S-W H\|)=\sqrt{1+\|S-W H\|^{2}}-1
$$

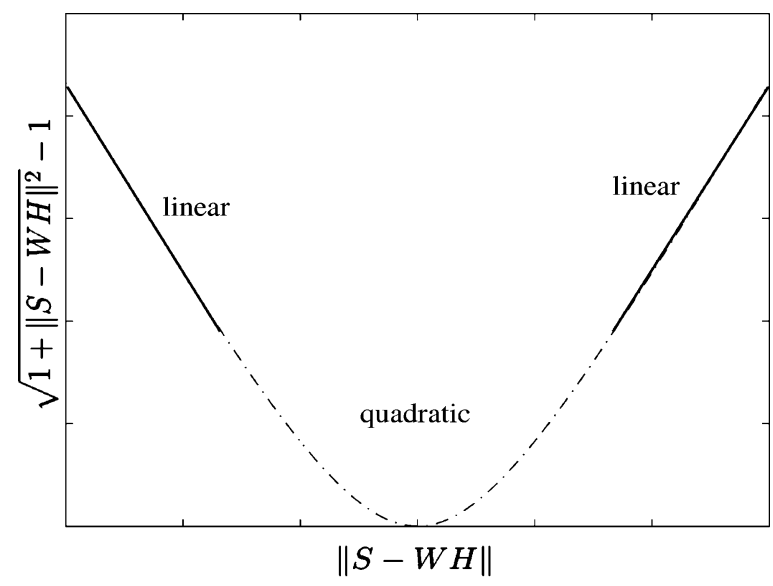

Fig. 1. Illustration of the hypersurface cost function.

which has two main attractive properties: i) it is quadratic when its argument is small and linear when its argument is large as illustrated in Fig. 1 and ii) its influence function $\varphi^{\prime}$ is differentiable and bounded. The influence function is a tool to describe the robustness properties of an estimator, and intuitively it measures the asymptotic bias caused by an infinitesimal contamination of the observations [12]. Unlike Huber influence function which is not differentiable [12], the smoothness of the hypersurface influence function is of great importance since it implies the continuity of its first derivative which in turn implies the continuity of the confidence intervals in the data points. This motivates us in introducing the RNMF approach, which is a result of minimizing the hypersurface cost function given by (3) subject to nonnegativity constraints on $W$ and $H$. The solution of this minimization problem is achieved using the steepest descent method and by updating alternatively two iterative equations as demonstrated in the next result.

Proposition 1: The minimization of the hypersurface cost function subject to nonnegativity constraints on $W$ and $H$ yields the following additive update rules:

$$
\begin{aligned}
W_{i k}^{(t+1)} & =W_{i k}^{(t)}-\alpha_{i k}^{(t)} \frac{\left(W H H^{T}\right)_{i k}^{(t)}-\left(S H^{T}\right)_{i k}^{(t)}}{\sqrt{1+\|S-W H\|}} \\
H_{k j}^{(t+1)} & =H_{k j}^{(t)}-\beta_{k j}^{(t)} \frac{\left(W^{T} W H\right)_{k j}^{(t)}-\left(W^{T} S\right)_{k j}^{(t)}}{\sqrt{1+\|S-W H\|}},
\end{aligned}
$$

where $\alpha_{i k}$ and $\beta_{k j}$ are the step sizes chosen at each iteration via Armijo rule for inexact line search.

Proof: To minimize the function $\varphi(W, H)$ defined as

$$
\begin{aligned}
\varphi(W, H) & =\frac{1}{2}\left(\sqrt{1+\|S-W H\|^{2}}-1\right) \\
& =\frac{1}{2}\left(\sqrt{1+\sum_{i j}\left(S_{i j}-(W H)_{i j}\right)^{2}}-1\right)
\end{aligned}
$$

we use an alternating method of steepest descent, that is the direction for which the function $\varphi$ will decrease the fastest. Let $W^{(t)}$ and $H^{(t)}$ denote the input values of the function $\varphi(W, H)$ at the $t$ th iteration. At each iteration, the function is updated alternatively as follows:

$$
\begin{aligned}
W_{i k}^{(t+1)} & =W_{i k}^{(t)}-\alpha_{i k}^{(t)} \frac{\partial \varphi(W, H)}{\partial W_{i k}^{(t)}} \\
H_{k j}^{(t+1)} & =H_{k j}^{(t)}-\beta_{k j}^{(t)} \frac{\partial \varphi(W, H)}{\partial H_{k j}^{(t)}}
\end{aligned}
$$




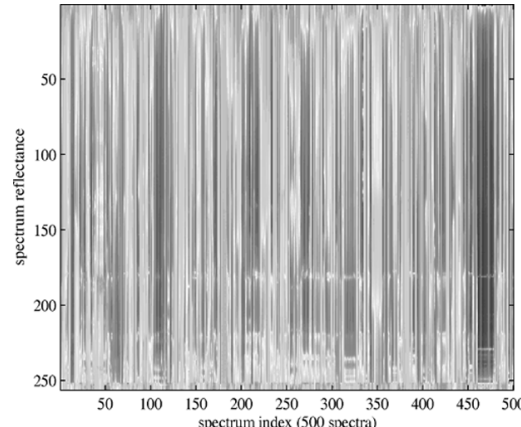

(a)

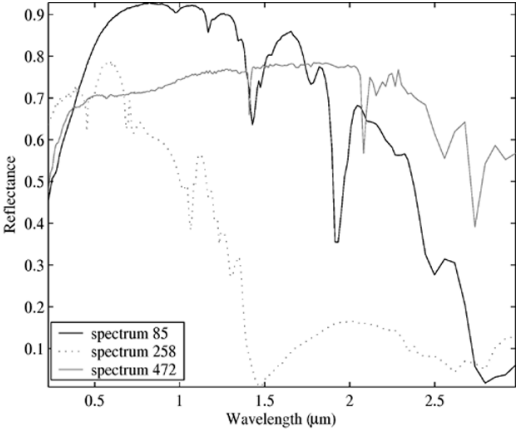

(b)

Fig. 2. (a) Image spectra and (b) samples of image spectra.

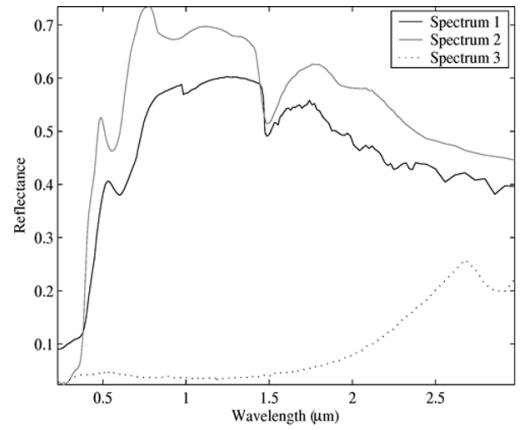

(a)

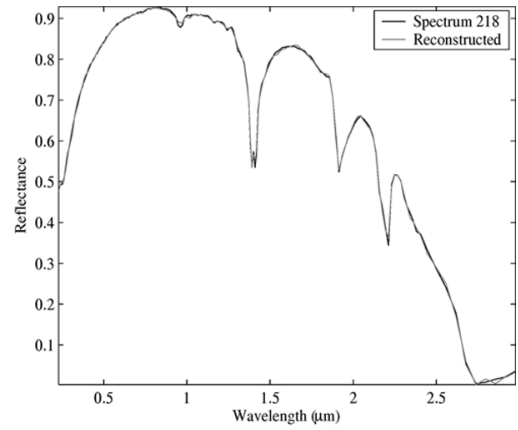

(b)

Fig. 3. PCA results: (a) top three principal components and (b) reconstruction of spectrum \#218 using 50 principal components.

where the matrices $W^{(0)}$ are $H^{(0)}$ are initialized as nonnegative random matrices, and $\alpha_{i k}, \beta_{k j}$ are scalars which determine the size of the step taken in the gradient direction, and are chosen at each iteration via Armijo rule for inexact line search [11]. The partial derivatives of $\varphi(W, H)$ with respect to $W_{i k}$ and $H_{k j}$ are given by

$$
\begin{aligned}
\frac{\partial \varphi(W, H)}{\partial W_{i k}} & =-\frac{\sum_{j} H_{k j}\left(S_{i j}-(W H)_{i j}\right)}{\sqrt{1+\|S-W H\|}} \\
& =-\frac{\left(S H^{T}\right)_{i k}+\left(W H H^{T}\right)_{i k}}{\sqrt{1+\|S-W H\|}} \\
\frac{\partial \varphi(W, H)}{\partial H_{k j}} & =-\frac{\sum_{i} W_{i k}\left(S_{i j}-(W H)_{i j}\right)}{\sqrt{1+\|S-W H\|}} \\
& =-\frac{\left(W^{T} S\right)_{k j}+\left(W^{T} W H\right)_{k j}}{\sqrt{1+\|S-W H\|}}
\end{aligned}
$$

After substitution we obtain the additive update rules. This completes the proof.

The goal of robust nonnegative matrix factorization is to determine meaningful basis functions for signals that are inherently nonnegative, and resistant to outliers. The idea is to approximate the original data by using a small number of basis functions combined with nonnegative coefficients, that is a nonnegative signal can be optimally encoded as a nonnegative additive combination of nonnegative basis functions. Hence, the RNMF algorithm is an iterative minimization algorithm, which modifies at each iteration the nonnegative basis functions (i.e., columns of $W$ ) and encodings (i.e., $H_{k j}$ ) until convergence.

\section{EXPERIMENTAL RESULTS}

In this section, we apply $\mathrm{K}-\mathrm{L}$ decomposition [2], nonnegative matrix factorization [4], [5], nonnegative sparse coding (NNSC) [8], nonneg- ative matrix factorization with sparseness constraints (NMFSC) [9], constrained nonnegative matrix factorization [19], and the proposed robust nonnegative matrix factorization method to a database of reflectance spectra. Denote by $S=\left\{\boldsymbol{f}_{j}: j=1, \ldots, n\right\}$ an $m \times n$ matrix of spectra, where each $\boldsymbol{f}_{j}$ (i.e., each column of $S$ ) is an $m$-dimensional vector representing the spectrum reflectance of $m$ samples, and $n$ is the number of spectra. Fig. 2(a) shows a $256 \times 500$ matrix of spectra $S$, which will be referred to as image spectra, and Fig. 2(b) depicts some samples of this image spectra. These spectra were collected from U.S. Geological Survey (USGS) Digital Spectral Library. The library includes samples of minerals, rocks, soils, physically constructed as well as mathematically computed mixtures, vegetation, microorganisms, and man-made materials. Each spectrum is a plot of the reflectance versus the wavelength as illustrated in Fig. 2(b), which displays three sample spectra of $S$ with index number (i.e., column number) 85,258 , and 472 .

\section{A. K-L Decomposition of Reflectance Spectra}

To test the K-L decomposition on the spectral data, we apply the PCA analysis on the USGS spectral library, which consists of 500 spectra. The results are illustrated in Fig. 3, where Fig. 3(a) shows the top three principal components of the reflectance spectra $S$. The reconstructed spectrum from 50 principal components is depicted in Fig. 3(b).

\section{B. Nonnegative Matrix Factorization of Reflectance Spectra}

The PCA technique is easy to implement, but the eigenvectors are not strictly positive basis functions and luck physical/intuitive meanings as illustrated in Fig. 3(a). The PCA factorization imposes no other constraints than the orthogonality, and hence allows the entries of $W$ and $H$ to be of arbitrary sign. Many basis images, for example, eigenfaces in 


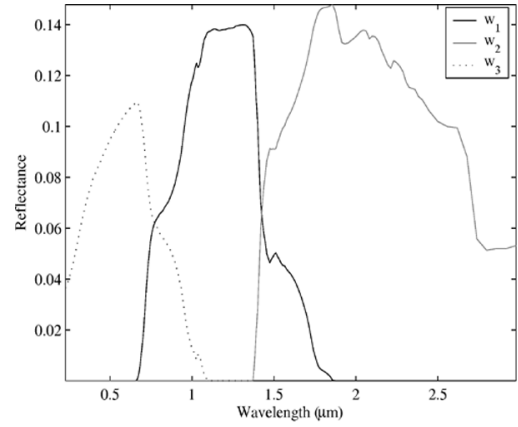

(a)

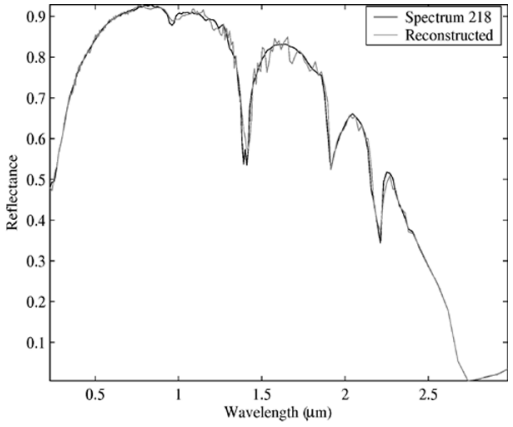

(b)

Fig. 4. NMF results: (a) first three NMF basis vectors and (b) reconstruction of spectrum \#218 using 50 basis vectors.

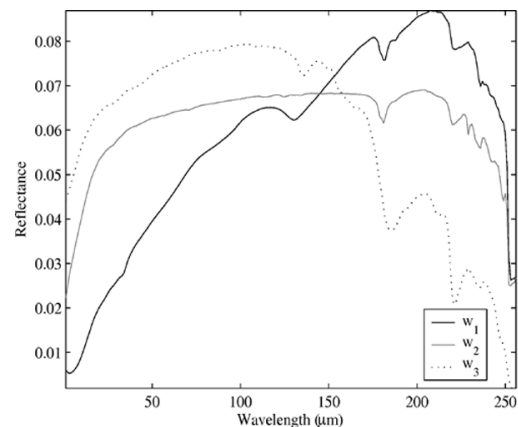

(a)

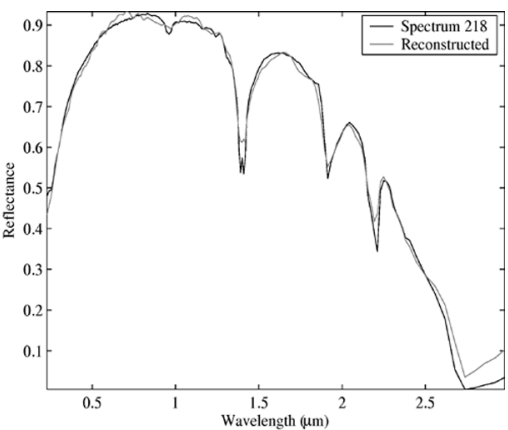

(b)

Fig. 5. NNSC results: (a) first three NNSC basis vectors and (b) reconstruction of spectrum \#218 using 50 basis vectors.

the case of face recognition, lack intuitive meaning; and a linear combination of them generally involves complex cancellations between positive and negative numbers [18]. The NMF allows only positive coefficients and thus nonsubtractive combinations. The NMF technique has been used with success in a variety of applications including text mining [6], and the identification/classification of nonimaging space objects [7].

Fig. 4(a) shows the first three basis vectors $\boldsymbol{w}_{j}$ of $S$ using nonnegative matrix factorization. Fig. 4(b) depicts the reconstructed spectrum from 50 basis vectors.

\section{Nonnegative Sparse Coding of Reflectance Spectra}

The goal of NNSC is to find a decomposition in which the hidden components (i.e., $\boldsymbol{h}_{j}$ ) are sparse, meaning that they have probability densities that are highly peaked at zero and have heavy tails [8]. This basically means that any given input vector can be well represented using only a few significantly nonzero hidden coefficients. This can formulated as a minimization problem, where the cost function to be minimized is given by $\mathcal{C}(W, H)=\|S-W H\|^{2}+\lambda \sum_{i j} H_{i j}$, subject to nonnegativity constraints on $W$ and $H$. The parameter $\lambda$ is given a priori or may be estimated, and it controls the tradeoff between the accurate reconstruction and the sparseness.

Fig. 5(a) shows the first three basis vectors $\boldsymbol{w}_{j}$ of $S$ using nonnegative sparse coding. Fig. 5(b) depicts the reconstructed spectrum from 50 basis vectors.

\section{Nonnegative Matrix Factorization With Sparseness Constraints}

The goal of NMFSC is to find a decomposition that will result in part-based representation [9]. This is done by incorporating sparseness constraints on the encoding and basis functions, that is we minimize the cost function $\|S-W H\|^{2}$ subject to the sparseness constraints $\operatorname{sparsness}\left(\boldsymbol{w}_{j}\right)=S_{w}$ and sparsness $\left(\boldsymbol{h}_{j}\right)=S_{h}$ for all columns $\boldsymbol{w}_{j}$ of
$W$ and rows $\boldsymbol{h}_{j}$ of $H$. The sparseness function of a vector $x \in \mathbb{R}^{n}$ is defined in terms of $L_{1}$ and $L_{2}$ norms as

$$
\operatorname{sparsness}(\boldsymbol{x})=\frac{\sqrt{n}-\|x\|_{1} /\|x\|_{2}}{\sqrt{n}-1} .
$$

Fig. 6(a) shows the first three basis vectors $\boldsymbol{w}_{j}$ of $S$ using NMFSC. Fig. 6(b)-(d) depicts the reconstructed spectrum from 50 basis vectors using different sparseness constraints as suggested in [9].

\section{E. Constrained Nonnegative Matrix Factorization}

The constrained nonnegative matrix factorization (cNMF) is a recent algorithm that was proposed for blindly recovering constituent source spectra from magnetic resonance chemical shift imaging of human brain [19]. This algorithm is formulated as

$$
\text { minimize }\|S-W H\|^{2} \text { subject to } H \geq 0 \text {. }
$$

Fig. 7(a) shows the first three basis vectors $w_{j}$ of $S$ using cNMF. Fig. 7(b) depicts the reconstructed spectrum from 50 basis vectors.

\section{F. Robust Nonnegative Matrix Factorization of Reflectance Spectra}

As discussed in the previous section, the robust nonnegative matrix factorization technique is resistant to outliers, and generates nonnegative basis functions which balances the logical attractiveness of measurement functions against their physical feasibility. The results of RNMF on reflectance spectra are depicted in Fig. 8, where Fig. 8(a) shows the first three basis vectors $\boldsymbol{w}_{j}$ of $S$ using robust nonnegative matrix factorization. Fig. 8(b) depicts the reconstructed spectrum from 50 basis vectors. This reconstructed spectrum, compared with the results obtained in the previous experiments, shows that the RNMF approach performs the best. The better performance evident from the 


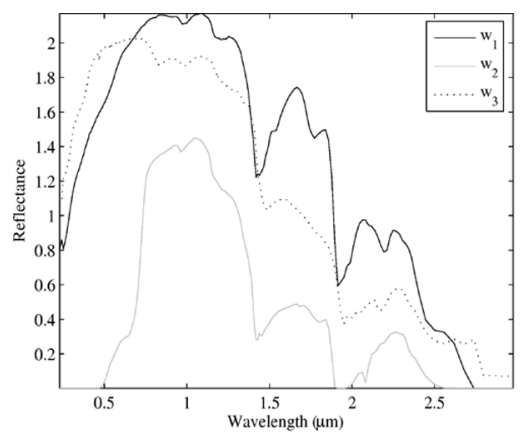

(a)

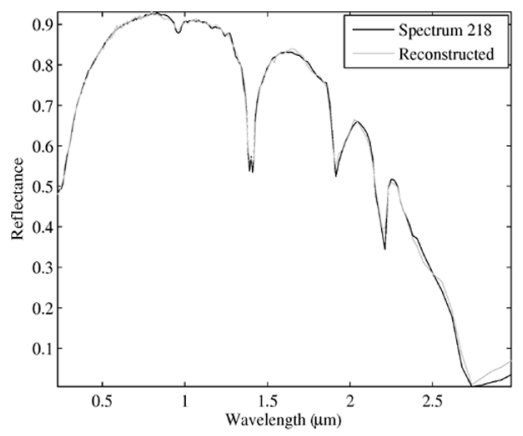

(c)

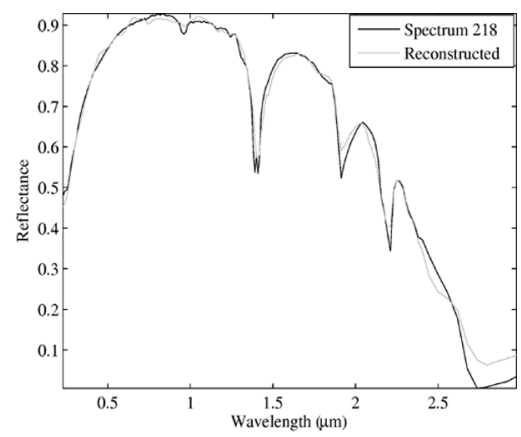

(b)

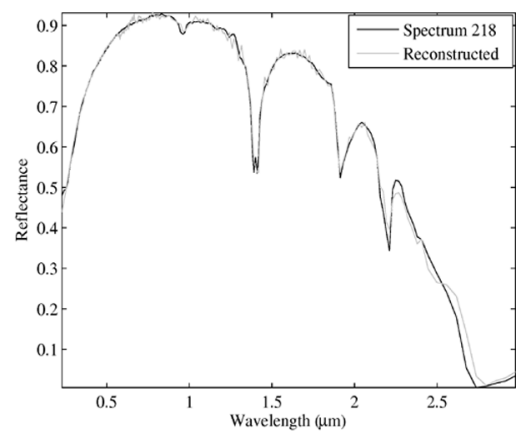

(d)

Fig. 6. NMFSC reconstruction of spectrum \#218 using 50 basis vectors: (a) first three NMFSC basis vectors and (b) spectrum reconstruction with $S_{h}=0.85$, and unconstrained $S_{w}$. (c) spectrum reconstruction with $S_{h}=S_{w}=0.5$, and (d) spectrum reconstruction with $S_{h}=0.8$ and unconstrained $S_{h}$.

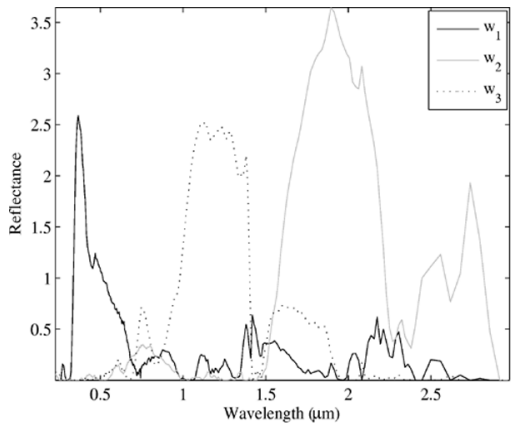

(a)

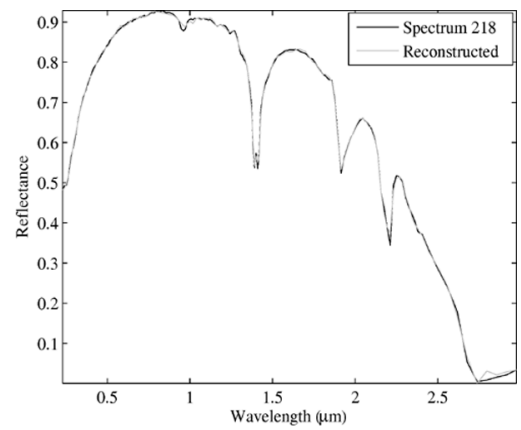

(b)

Fig. 7. cNMF results: (a) first three cNMF basis vectors and (b) reconstruction of spectrum \#218 using 50 basis vectors.

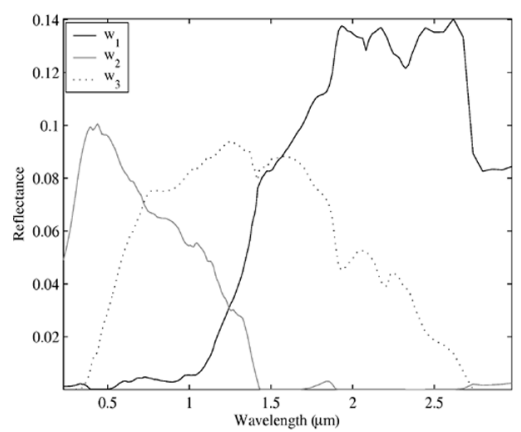

(a)

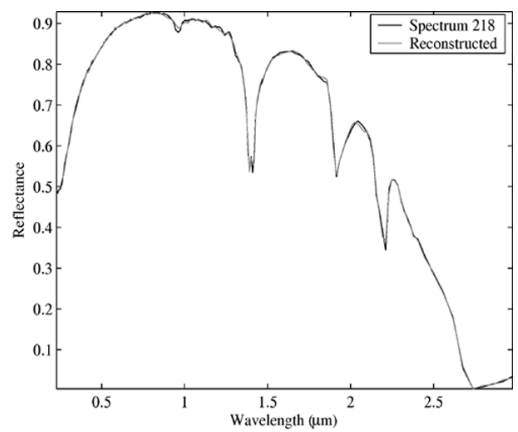

(b)

Fig. 8. RNMF results: (a) first three RNMF basis vectors and (b) reconstruction of spectrum \#218 using 50 basis vectors.

RNMF approach as depicted in Fig. 8(b) is in fact consistent with a variety of spectra used for experimentation. In addition, the relative error versus the iteration number as illustrated in Fig. 9 clearly demon- strates the convergence of the RNMF method using as a stopping criterion $\left\|(W H)^{(k+1)}-(W H)^{(k)}\right\|_{2} /\left\|(W H)^{(k)}\right\|_{2}<\epsilon$, where $\epsilon$ is sufficiently small, and $k$ denotes the $k$ th iteration. 


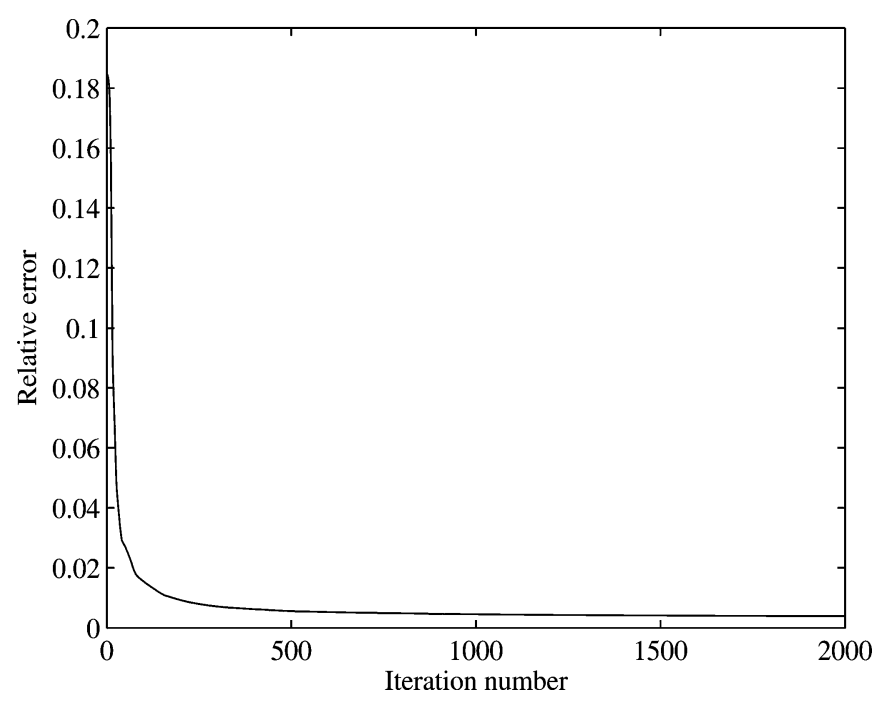

Fig. 9. RNMF convergence: Relative error versus Iteration number.

\section{CONCLUSION}

In this paper, we proposed a robust approach to nonnegative matrix factorization of a spectral library. The proposed method is formulated as an energy minimization problem whose solution is achieved by updating alternatively two equations. Unlike the $\mathrm{K}-\mathrm{L}$ method, the robust nonnegative matrix factorization technique is based on a robust cost function, resistant to outliers, and generates nonnegative basis functions which balances the logical attractiveness of measurement functions against their physical feasibility. We have successfully tested the robust nonnegative factorization algorithm on a library of reflectance spectra, and the experimental results clearly show that the proposed technique outperforms the current reconstruction methods including PCA, NNSC, NMFSC, NMF, and cNMF.

\section{REFERENCES}

[1] J. M. Chalmers and P. R. Griffiths, Handbook of Vibrational Spectroscopy. New York: Wiley, 2002.

[2] I. T. Jolliffe, Principal Component Analysis. New York: SpringerVerlag, 1986.

[3] K. V. Mardia, J. T. Kent, and J. M. Bibby, Multivariate Analysis. London, U.K.: Academic, 1979.

[4] D. Lee and H. Seung, "Learning the parts of objects by nonnegative matrix factorization," Nature, vol. 401, pp. 788-791, 1999.

[5] —_, "Algorithms for nonnegative matrix factorization," Adv. Neural Inf. Proc. Syst., vol. 13, pp. 556-562, 2000.

[6] F. Shahnaz, M. Berry, V. P. Pauca, and R. Plemmons, "Document clustering using nonnegative matrix factorization," Information Processing and Management, vol. 42, no. 2, pp. 556-562, Mar. 2005.

[7] J. Piper, V. P. Pauca, R. Plemmons, and M. Giffin, "Object characterization from spectral data using nonnegative factorization and information theory," presented at the Amos Technical Conf., Maui, Sep. 2004.

[8] P. O. Hoyer, "Nonnegative sparse coding," in Proc. IEEE Workshop Neural Networks Signal Processing, Sep. 2002, pp. 557-565.

[9] —, "Non-negative Matrix Factorization with sparseness constraints," J. Mach. Learn. Res., no. 5, pp. 1457-1469, 2004.

[10] C. Samson, L. Blanc-Féraud, G. Aubert, and J. Zerubia, "A variational model for image classification and restoration," IEEE Trans. Pattern Anal. Mach. Intell., vol. 22, no. 5, pp. 460-472, 2000.

[11] C. T. Kelley, Iterative Methods for Linear and Nonlinear Equations. Philadelphia, PA: SIAM, 1995, vol. 16, Frontiers in Applied Mathematics.

[12] P. Huber, Robust Statistics. New York: Wiley, 1981.

[13] F. R. Hampel, E. M. Ronchetti, P. J. Rousseeuw, and W. A. Stahel, Robust Statistics: The Approach Based on Influence Functions. New York: Wiley, 1986.
[14] M. J. Black and A. Rangarajan, "On the unification of line processes, outlier rejection, and robust statistics with applications in early vision," Int. J. Comput. Vis., vol. 19, pp. 57-92, Jul. 1996.

[15] M. J. Black, G. Sapiro, D. H. Marimont, and D. Heeger, "Robust anisotropic diffusion," IEEE Trans. Image Process., vol. 7, no. 3, pp. 421-432, Mar. 1998

[16] H. Krim and I. C. Schick, "Minimax description length for signal denoising and optimized representation," IEEE Trans. Inf. Theory, vol. 45, no. 3, pp. 898-908, Apr. 1999.

[17] A. B. Hamza and H. Krim, "Image denoising: a nonlinear robust statistical approach," IEEE Trans. Signal Process., vol. 49, no. 12, pp. 3045-3054, Dec. 2001.

[18] M. Turk and A. Pentland, "Face recognition using eigenfaces," in Proc. IEEE Conf. Computer Vision and Pattern Recognition, 1991, pp. 586-591.

[19] P. Sajda, S. Du, T. R. Brown, R. Stoyanova, D. C. Shungu, M. Xiangling, and L. C. Parra, "Nonnegative matrix factorization for rapid recovery of constituent spectra in magnetic resonance chemical shift imaging of the brain," IEEE Trans. Med. Imag., vol. 23, no. 12, pp. 1453-1465, Dec. 2004.

\section{Low-Complexity Equalization of Time-Varying Channels With Precoding}

Zijian Tang, Student Member, IEEE, and Geert Leus, Senior Member, IEEE

Abstract-This correspondence deals with time-varying (TV) single-input multiple-output (SIMO) channels, which are both frequency selective (due to high data rate) and time selective (due to mobility). A complex exponential basis expansion model (CE-BEM) is used to model the channel. We consider a block transmission system, where on the transmit side a precoder is employed to enable the maximum available diversity for a CE-BEM channel. After direct decoding on the receive side, the resulting channel resembles a finite-impulse-response (FIR) filter on both block and symbol level. We therefore propose an equalizer that bears a structure analogous to the effective channel. In comparison with a standard block minimum mean-square error decision-feedback equalizer (BMMSE-DFE) that involves the inversion of a large-size matrix, the proposed parametric equalizer renders a similar performance but at a lower computational cost if there are multiple outputs present. Another contribution of this correspondence is a semiblind algorithm to estimate this equalizer when the channel state information is not available: the equalizer taps and the information symbol estimates are refined recursively by means of normalized least-mean-squares (NLMS) adaptation.

Index Terms-Basis expansion model (BEM), diversity, time-varying (TV) channel.

\section{INTRODUCTION}

In high-data-rate mobile communication systems, the relative velocity between the transmitter and the receiver gives rise to a Doppler

Manuscript received September 15, 2005; revised November 17, 2005. The associate editor coordinating the review of this manuscript and approving it for publication was Dr. Nicholas D. Sidiropoulos. This research was supported in part by NWO-STW under project DTC.5893 (VICI-SPCOM) and DTC. 6577 (VIDI-SPCOM). This work was presented in part at the IEEE Workshop on Signal Processing Advances in Wireless Communications (SPAWC), New York, June 2005.

The authors are with the Delft University of Technology-Faculty of EWI/ Electrical Engineering, 2628 CD Delft, The Netherlands (e-mail: tang@ cas.et. tudelft.nl; leus@cas.et.tudelft.nl).

Digital Object Identifier 10.1109/TSP.2006.879261 